\title{
Supporting the entire life-cycle of the extended manufacturing enterprise
}

\author{
Americo Azevedo $^{\mathrm{a}, \mathrm{b}, *}$, José Faria ${ }^{\mathrm{a}, \mathrm{b}}$, Filipe Ferreira ${ }^{\mathrm{a}}$ \\ a CESE/INESC TEC, Porto, Portugal \\ ${ }^{\mathrm{b}}$ Faculdade de Engenharia, Universidade do Porto, Rua Dr. Roberto Frias, Porto 4200-465, Portugal
}

\section{A R T I C L E I N F O}

\section{Article history:}

Received 17 April 2015

Received in revised form

14 May 2016

Accepted 16 May 2016

Available online 25 May 2016

Keywords:

Extended manufacturing enterprise

One-of-a-kind manufacturing

Engineer-to-order environment

Manufacturing reference model

Manufacturing life-cycle framework

\begin{abstract}
A B S T R A C T
This paper presents a framework to support the full life-cycle of extended manufacturing enterprises, from creation to operation and dissolution phases. The deployment and operation of such enterprises can be compared with the concept of 'plug-and-play', as the internal processes and legacy systems of the companies involved are smoothly integrated within an overall business process designed, validated and executed according to a specific business opportunity. During the plug phase, the specific business requirements are elicited and integrated with the design of the extended business processes. On the other hand, in the play phase, those predefined processes are executed in order to run the extended enterprise successfully. The paper describes an application case regarding an engineer-to-order and one-of-a-kind engineering product. This scenario is common to a large number of technology-driven SMEs, and illustrates the value of the framework to exploit business opportunities that require a combination of skills and resources that do not exist in-house. The case shows how the platform addresses the two main challenges in the deployment of an extended enterprise. The first challenge is finding the right set of partners to address a new business opportunity and the design of the underlying collaborative processes. The second challenge is mostly technical, and focuses on the integration of the legacy systems of the partners participating in the network so that cooperation can take place quickly and seamlessly.
\end{abstract}

(c) 2016 Elsevier Ltd. All rights reserved.

\section{Introduction}

Nowadays, enterprises seek to adopt new business models and to explore new organisational models, such as dynamic networks and virtual enterprises, to quickly respond to market opportunities while assuring fast time to market, a differentiated offer and competitive prices [1]. Furthermore, business-manufacturing sustainability will focus more and more on high-variety, low-volume and quick delivery businesses models associated with complex products [2] which require a combination of multiple skills that, in an SME, may not exist in-house. Over the last decade, several research initiatives and projects tried to address the needs and requirements of such emerging business models. However, in spite of this effort, currently there are no proven or effective methodologies, approaches or tools to support the enterprises in creating, managing and participating in the collaborative networks inherent to this type of business models (e.g. dynamic networks and virtual enterprises) $[3,4]$.

\footnotetext{
* Corresponding author at: CESE/INESC TEC, Campus da FEUP, Rua Dr. Roberto Frias, 4200-465 Porto, Portugal.

E-mail address: ala@fe.up.pt (A. Azevedo).
}

Collaborative networks in industry come in a large variety of forms. Moving from the classical supply chains format, characterised by relatively stable networks with well-defined roles and requiring only minimal coordination and information exchange, more dynamic structures are emerging in industry. Some of these organisational forms are goal-oriented, that is, focused on a single project or business opportunity, such as the case of virtual enterprises [5].

Manufacturing networks and "Virtual Enterprises" have been widely discussed over the past decade $[15,16]$. In this research, the definition of virtual enterprise considered is based on the definition proposed by Camarinha-Matos [5], which states: a Virtual Enterprise represents a temporary alliance of enterprises that come together to share skills or core competences and resources in order to better respond to business opportunities, and whose cooperation is supported by computer networks. A "Virtual Manufacturing Enterprise" is one form of Collaborative Networked Organisations [6] that allows partners to exploit emerging business opportunities in a flexible way. An "Extended Enterprise" is viewed as a particular case of a virtual enterprise. It occurs when an organisation "extends" its boundaries to all or some of its suppliers, which is the case of several innovative SMEs in the manufacturing industry.

In the context of these research topics [6], several categories of 
goal-oriented collaborative networks have been identified and defined, and four of them are grasping opportunity-driven and naturally relevant in the scope of this research targeting the collaborative manufacturing domain [4]. These grasping opportunitydriven Collaborative Networked Organisations are formed to meet a specific business opportunity, and will dissolve once their mission is accomplished. The concept of Extended Enterprise is also relevant for this research.

The effective implementation of a virtual organisation raises several challenges: the integration of human and technical resources to enhance workforce performance and satisfaction; the need to instantaneously transform information gathered from a vast array of sources into useful knowledge for making effective decisions; the ability to reconfigure manufacturing enterprises rapidly in response to changing needs and opportunities; and developing innovative manufacturing processes and products with a focus on decreasing dimensional scale.

Those challenges may be grouped in two main issues. The first is a business-oriented challenge where the goal is to find the right set of partners to address a new business opportunity and the deployment of the collaborative processes. The second is a mostly technical challenge, and the goal is to integrate the legacy information systems of the partners involved in the collaborative network so that cooperation can take place quickly and seamlessly.

This paper presents a framework that addresses these two main issues. The business issues comprise a reference model with a catalogue of business processes and practical approaches. To enable the technical integration issues of the extended manufacturing enterprise, the framework provides a technical platform offering several tools and mechanisms to leverage the exchange of information between factories at a manufacturing process level. The practical application example provided in the final part of the paper addresses these two issues in order to make it easier to understand the applicability of the framework to develop collaborative business opportunities in an engineer-to-order and oneof-a-kind production SME.

\section{The plug and play virtual factory concept}

The concept of combining the power of several independent business entities to implement complex supply chain processes, such as the so-called virtual manufacturing enterprises, is not new and has been addressed by several research projects in recent years [3,5,6,19]. However, most of them are limited to creating virtual manufacturing enterprises at business level, and in many cases they concentrate on the partner-finding and factory-building stages. To this date, no proven tools and technologies exist on the market to provide valuable end-to-end integrated Information and Communication Technology (ICT) in such environments [3]. Thus the current approaches do not allow for innovation and higher management and operational efficiency in networked operations and framework. The platform presented here aims to change this situation [4].

The framework introduced in the next section addresses the integration, interoperability, and collaboration challenges within supply chains, lowering market access barriers for SMEs, which usually neither have the capabilities to control the entire manufacturing life-cycle nor the market power to enforce their own interfaces and standards. This way, it will be easier for SMEs in the manufacturing industry to engage in collaboration networks in the context of virtual organisations.

Two different interoperability approaches were envisioned: one based on a hard integration between the partners' legacy systems and the management platform of the extended enterprise, and a soft integration approach allowing the participation in the extended enterprise through a cloud-based web interface. The first approach is suitable for larger companies with sophisticated management systems and capable of dealing with the technical integration of IT systems. The cloud-based approach may be more interesting to small companies with limited resources

The platform is based on the paradigm of Service-oriented Architectures (SOA), exploiting latest technologies from the "internet of things", and leveraging recent developments in the area of cloud computing. This way, the platform facilitates the participation of SMEs in large ad-hoc supplier/customer networks, providing the tools and methods to tackle the challenges described above and improving information exchange and sharing with the other partners of the virtual manufacturing enterprise [4]. The platform provides tools and methods to design, simulate, optimise, execute, adapt and monitor dynamic manufacturing processes through a web-based dashboard (see Fig. 1). The SOA manufacturing approach leads to the description of each virtual enterprise process step as a service $[7,8]$ and allows manufacturing companies to model and manage their processes as a composition of distributed and heterogeneous services.

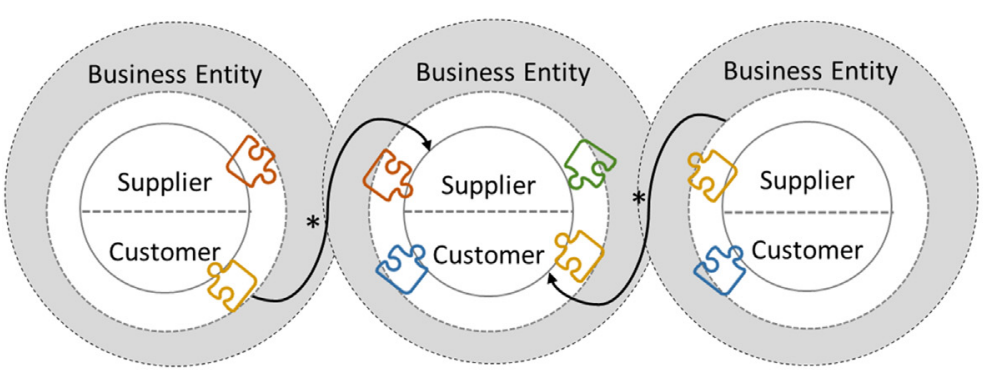

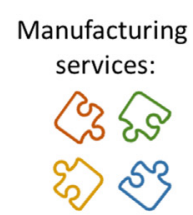

a)

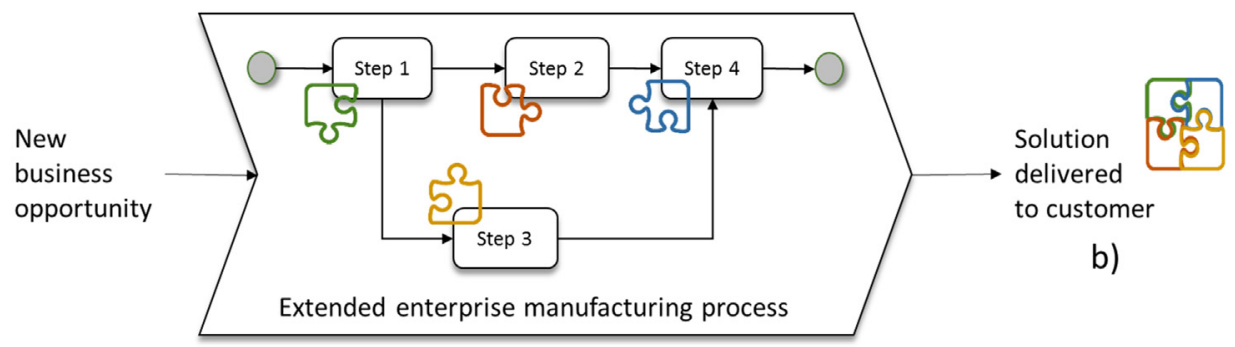

Fig. 1. The plug-and-play manufacturing concept. 


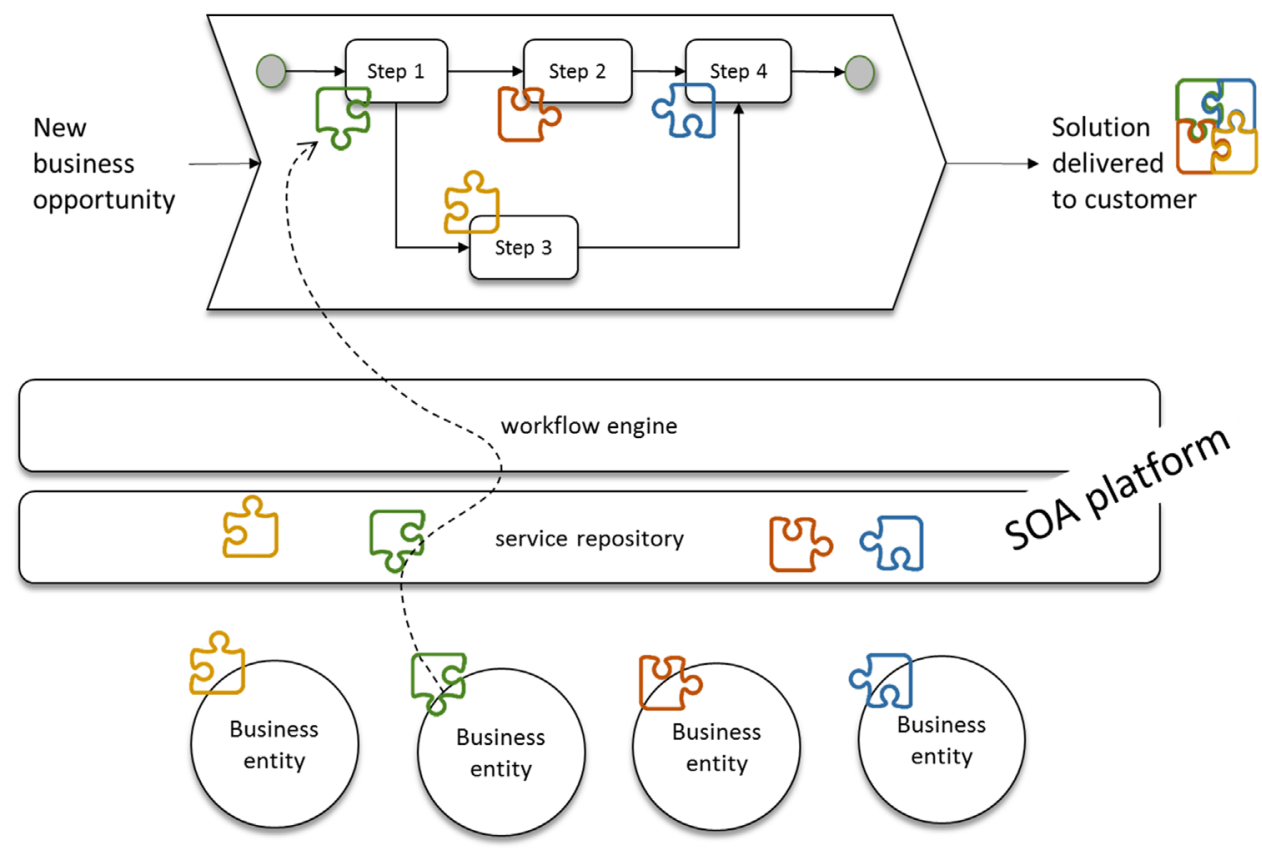

Fig. 2. The SOA platform concept.

In the context of a virtual manufacturing enterprise, each business entity participating in the virtual chain may act both as a provider and as a consumer of services.

The typical applications for the framework are characterised by the manufacturing of complex products, a one-of-a-kind environment and the involvement of multiple business partners. In this kind of environment, for each customer order, there are specific engineering and production activities which should be managed as a whole. For each customer order, there is a mix of internal and external orders. Internal orders are usually managed by existing legacy systems, but the external orders are not. Both engineering and production activities could be outsourced (in each customer order, several activities could be outsourced).

As represented in Fig. 1, each business entity participating in the virtual enterprise may act as a provider, as well as a consumer of manufacturing services. The way these entities collaborate in order to create value is described using a business process model, as exemplified in Fig. 1(b).

There is an increasing usage of SOA technologies in industrial applications [20,21,24]. In fact, as discussed in [22], SOA seems to be the most effective approach to lead loosely coupled applications, which is the case of extended enterprises.

When a new business opportunity is originated, only a rough design exists of the process instance that will deal with that opportunity. The process design will then be progressively refined and adapted mostly based on human judgement and decision (human-based flexible and adaptive processes) [9]. Another distinctive feature of this type of virtual enterprises is the fact that they involve product design, process engineering and manufacturing tasks. All these tasks have to be managed comprehensively by the virtual manufacturing enterprise management system. Also, several documents are created during execution time, such as customer orders, engineering drawings, production and quality records, and after-sales tickets. These documents are spread over various systems and supports (for instance, paper, electronic, $\mathrm{CAD}$, text processor office files), but even so they have to be fully traceable.

Another major challenge in the design of such networked manufacturing environments is estimating operational performance [17] and production planning, order splitting and order assignment, taking into account production time window and capacity [18].

Currently, there is no single solution that meets the functional and technical requirements for this type of environment. Very often, the collaborative manufacturing process is mainly managed ad-hoc, and although partners are chosen on the basis of the qualification process, when it is necessary to collaborate, partners engage in bilateral interactions. The entire process is managed manually using email, phone calls and physical meetings.

During process execution, human judgement and decision play a central role when unexpected events occur. To know the status of each task the process manager has to contact the local responsible at each partner. This way, much information is kept in peoples' minds and is never registered, thus originating avoidable errors and delays in the delivery of the final product.

Moreover, if the partners' legacy systems do not comply with a common standard, system integration throughout the supply chain will require multiple one-to-one interfaces, and the complexity of the management system will increase exponentially.

With an SOA-based platform, the approach is quite different. Each partner publishes its services in a shared repository, and according to a common specification standard. When a new business opportunity occurs, a dedicated process model is designed in order to address its specific requirements. To orchestrate the execution of the process, the workflow engine invokes the appropriate services on the partners' legacy systems using the standard interfaces, as depicted in Fig. 2. This way, the plug-andplay virtual enterprise is expected to facilitate the set-up of the collaboration networks and their operation, adaption, assessment and improvement in a faster and more effective way.

Two integration approaches were devised in order to support the seamless interoperability of companies with different IT maturity levels within the same virtual enterprise. In fact, the ADVENTURE framework is able to handle partners willing to participate in a virtual factory with different levels of integration. As SMEs may not have the capacity to implement innovative manufacturing technologies, the system must be highly adaptable to any equipment with minimum integration and setup requirements [23]. While large companies may implement hard integration solutions, small SMEs will be able to participate using browser or mobile interfaces. To 
support this business need, three components were developed: Customer Web Portal, Supplier Web Portal and a Mobile Application. These components support the interactions, as well as the exchange of data, documents and messages between the small SMEs.

\section{The extended enterprise life-cycle}

The extended manufacturing enterprise life-cycle is triggered by an emerging business opportunity and encompasses the following main activities described hereafter: analysis, design, execution and adaptation, improvement and dissolution.

The process analysis activity refers to a preliminary business opportunity analysis where it is decided whether an opportunity is reasonable and profitable or not. In this activity, the objectives, boundaries, inputs, outputs and stakeholders of the potential processes, as well as the performance measurements and recommendations, are defined and developed, respectively.

The design activity includes tasks for process definition, partner search and assignment, process simulation and process optimisation. The user is able to define a new extended manufacturing process using the smart process definition tool. Then, the partners to be assigned to each of the process activities can be searched by using the Data Provisioning and Discovery module. Once partners have been assigned to the manufacturing process activities, the process simulation plug-in can be used in order to obtain the results of a given process configuration. If the results are not satisfactory, it is possible to use the optimisation component to achieve the best global results for the manufacturing process. Thus, by applying optimisation algorithms, this component makes it possible to optimise partner service assignment, satisfying constraints and minimising or maximising the objective function defined.

The execution and adaptation activities include tasks for process execution, monitoring, and adaptation. When the process is triggered, a workflow engine will manage the sequence of activities and invoke the associated manufacturing services. The monitoring module is then used to obtain in-depth information regarding relevant process task data. This module will include a rules engine and will be responsible for triggering alerts and notifications. Information from legacy systems and wireless sensors will be available by means of a technical integration. This way, a real-time process monitoring is achieved.

The improvement and dissolution activities refer to the evaluation of the executed process and to the enquiry of members. All the achievements (information, knowledge and experience) are stored in a common, cloud-based repository, which enables future enterprises to profit from this enhanced knowledge base.

It should be noted that the set of reference business processes which will be used as a basis for defining the different processes of operationalising the business model of the Extended Manufacturing Enterprise - will play a fundamental role in the ADVENTURE framework. In fact, the concept of business process is becoming increasingly important and has been widely accepted as central to the operations of an organisation, namely to understand how it creates value for its customers. This paper adopts the definition of Weske [10] for business process: "A business process consists of a set of activities that are performed in coordination in an organisational and technical environment. These activities jointly realise a business goal."

The studies performed on certain industry or domain-specific areas, as well as the domain requirements analysis performed, make it possible to conclude that some available frameworks (such as SCOR [12] and VRM [13]) will potentially be a foundation of the reference model to be considered in the framework (this reference model is presented in the next section). Furthermore, we are also looking for other reference frameworks of business processes, namely the ones resulting from research projects in same or close research domains (e.g. NetChallenge project [3]).

The following image presents the mapping between the extended enterprise life-cycle and some relevant elements of the framework's reference model (Fig. 3).

The first stage, Join, encompasses the adoption of the technological platform, its implementation and integration with other enterprise systems and cyber physical environments, as well as the provision of data in the cloud services database. The second stage, Plug, is triggered by a new business opportunity. Here, an analysis of the needs is conducted, the search and assignment of partners is initiated, and the procedures are defined. The Play stage corresponds to the process execution, adaption, where the product is manufactured and delivered to the customer. After a successful delivery, the Dissolution stage takes place, including partner performance evaluation, sharing of benefits and conclusion of the process.

\section{The framework}

The framework was developed in the context of the ADVENTURE research project (ADaptive Virtual ENterprise ManufacTURing Environment) [14], hereafter referred to as the ADVENTURE framework.

During the process analysis activity, the new business opportunity and the process adaptation needs are examined so that it is possible to create an abstract model of the extended manufacturing enterprise processes. The objectives, boundaries, inputs, outputs, and stakeholders of the potential manufacturing Processes are defined, as well as the performance measurements and recommendations. At the end of this activity, the broker has gathered all the information necessary to decide if the collaborative manufacturing process should be implemented or not.

In order to support the analysis activities, a reference model denoted as ARM (Adventure Reference Model) was developed. It consists of a business development methodology, a catalogue of business processes and a catalogue of best practices. These tools and guidelines are available in the Knowledge and Information Repository, where it is possible to conduct searches and submit queries in order to obtain information about similar processes that

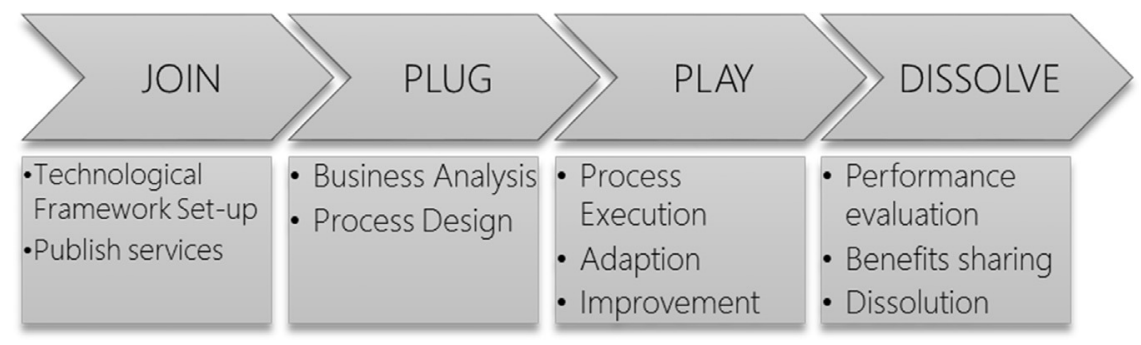

Fig. 3. Mapping between extended enterprise life-cycle and the framework's reference model. 
have been executed in the past. Using the integrated partner search engine, managers are able to search partners and filter them by industry type, location, or work load level, for instance. Furthermore, some preliminary simulations can be done in order to decide whether an opportunity is reasonable and profitable or not.

The overall structure of the ARM reference model is presented in Fig. 4. The set of activities of the life-cycle are organised according to four main phases: JOIN, PLUG, PLAY, and DISSOLVE. Each phase has several processes and each process comprises several management practices that may be adopted. The ARM reference model describes each process by providing the inputtransformation-output model and the sequence of its activities in a BMPN diagram. Furthermore, it provides for each process a description of inputs, outputs, practices and how the platform enables it. A detailed description of the reference model can be found in [14].

Fig. 5 depicts the process model, as well as the practices associated with the activity "Set-up Adventure framework".

The framework also includes a technological platform comprising several tools to support the other activities (design, simulation, optimisation, execution, adaption and dissolution) of the life-cycle, as described in the following paragraphs.

During the design activity, the abstract model of the extended business process is developed, which describes the entire manufacturing process envisioned, as well as the services required in terms of resources and manufacturing capabilities offered by real factories. Using the smart process definition tool, the manufacturing processes of the extended enterprises are designed following a service-oriented approach, including constraints, such as environmental and ethical questions, lead time, and costs. Partners are searched according to manufacturing capability descriptions, available in the knowledge and information repository, including

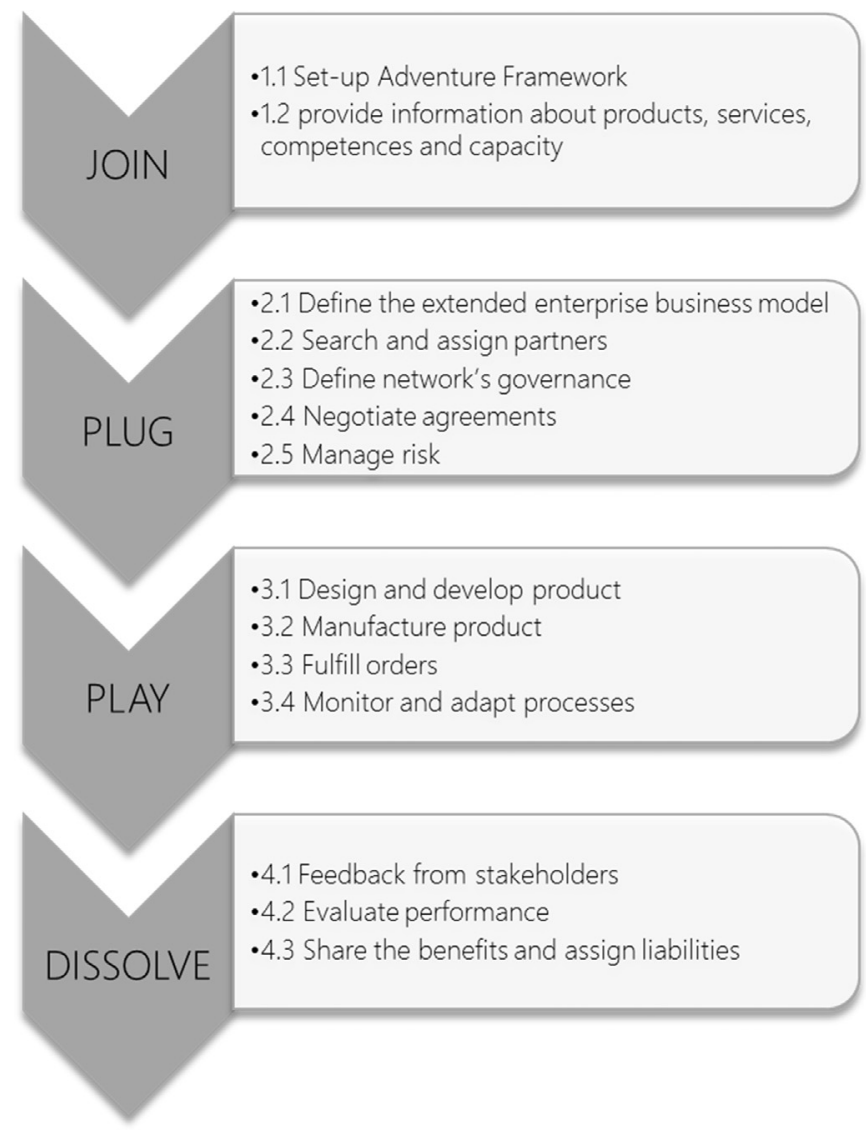

Fig. 4. Overall organisation of the Reference Model. delivery time, quality and technical requirements. Partners are then assigned to each of the process activities or sub-processes.

Once the process is completely defined, the simulation component can be used in order to evaluate whether all requirements are satisfied. Otherwise, optimisation techniques can be applied to the modelled Manufacturing Processes in order to make sure that the requirements are met, and the process is efficiently executed. These optimisation techniques include support for achieving an optimised selection and assignment of partners to respective process steps, as well as propositions for rearranging and restructuring the modelled Manufacturing Processes. If all requirements are fulfilled during the simulation and forecasting, it is possible to start negotiating contracts with eligible candidate partner factories in order to achieve the best results.

During the execution and adaptation phase, the designed Manufacturing Process will be executed and adapted if necessary. The best-fitting and contracted partner factories, identified and selected during the design phase, are employed and the modelled Manufacturing Process is executed and monitored. While the actual execution takes place, the monitoring component collects and provides real-time information about the current status of the Manufacturing Process. Unforeseen events may either be recognised by this monitoring component or by (intermediate) products or product parts enhanced with monitoring and error detection capabilities. Information from the different ICT systems and sensors of the customers, suppliers and shipment partners, as well as from human input, is also integrated into process monitoring.

The process status information is prepared in such a way that there will be no difference between executing and monitoring a company-internal or cross-organisational manufacturing process.

If the monitoring shows unforeseen events which might change the outcome of the process with regard to deadlines, costs or other criteria, or if something different goes wrong with the contracted suppliers during the process runtime, an automatic alert will be triggered indicating necessary adaptations to the process, for instance redirecting distinct manufacturing steps to other partners or exchanging underperforming, unsatisfying partners. The selection of alternative or complementary candidate factories and their manufacturing services, respectively, can either be performed manually or can automatically be assisted and performed by the Process Optimisation component, which automatically creates a list of best matching partners and proposes this list to the process manager. The system thereby lets the process manager make the final decision or automatically selects the best fitting suppliers depending on their preferences.

Also, if the process requirements change, for instance because more products are needed or different features of a product are mandated, the process adaptation or the optimisation option described above will be presented automatically.

The improvement activities take place when the extended manufacturing enterprise life-cycle reaches its end, and an evaluation takes place in the improvement phase. For this, the process manager agrees with the partners, who have participated in the extended enterprise, on evaluating the executed process. The reference models can be improved and lessons learnt are stored in the Knowledge and Information Repository so that future users of the platform can access them.

Thus, in the improvement activity, information from the extended manufacturing enterprise, such as best practices and lessons learnt are created (and stored) so that a user of the platform can query the Knowledge and Information Repository in order to obtain information about manufacturing processes that have been executed in the past, (probably) applying retrieved practices and avoiding mistakes which have been identified. This way, the platform builds a knowledge base which is enriched and updated 


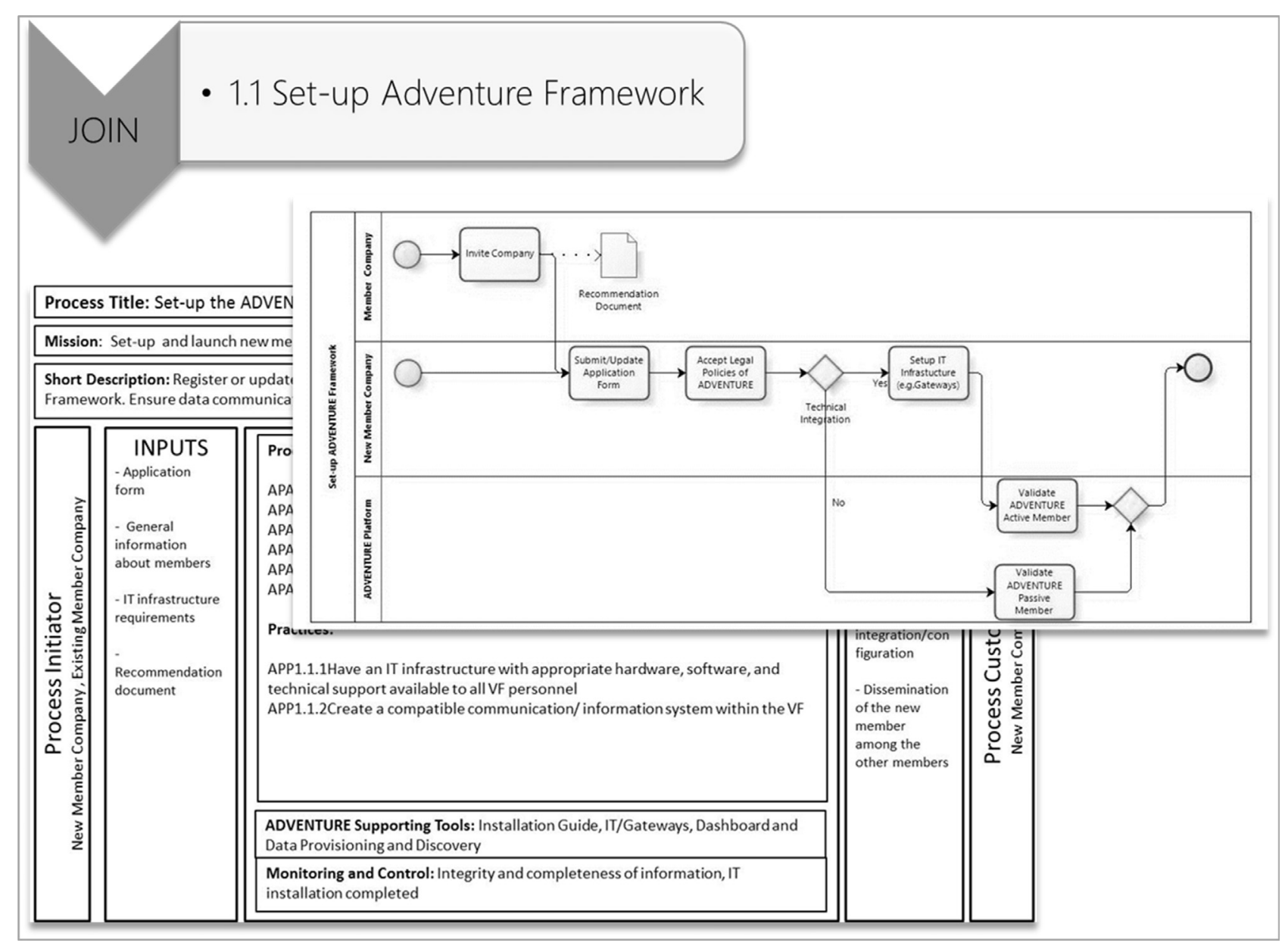

Fig. 5. Process Description and Best Practices Sheet (activity: set-up Adventure framework).

each time a Process is executed.

After having updated the Knowledge and Information Repository, the extended enterprise is finally dissolved. However, as all the information and configurations of the dissolved extended manufacturing enterprise have been stored, it is possible to reinitiate the extended manufacturing process - conducting adaptations based on lessons learnt which have been stored during former improvement phases - in case the same product has to be manufactured again.

The main modules of the platform that support the life-cycle activities are depicted in Fig. 6.

\section{Business application case}

The application case discussed hereafter relates to a leading machinery manufacturer specialised in technology, targeting the cork transformation industry. The company develops, produces, sells and provides after sales assistance to a wide range of production machinery. Products are complex due to the fact that they are comprised of hundreds of different components and different technologies (multi-part and multi-technology products associated with converging structures). To manufacture these products, several process and assembly technologies are involved, namely casting, bending, milling, welding, painting, sub-assembly and assembly. Moreover, various automation technologies and components are involved as well, namely automation controllers, advanced sensing devices, image processing, electric and pneumatic servo-drives technologies.
The company only outsources activities that are not covered by in-house competences such as bending, casting, part coating treatment and, on very few occasions, specific product engineering. The main reasons for outsourcing are the lack of competences (mainly technological) in some operations and the increase of general factory productivity by reducing delivery time to customers.

In fact, some products consist of up to thousands of different components. This fact implies a vast amount of documentation (related to customer interactions, product engineering, process definition, industrialisation, quality, after sales assistance) and data (production, quality records), which has to be created and maintained. Documentation and data are spread in different physical locations at the plant, in different formats (paper and/or electronic) and also in different software frameworks (CAD, Microsoft Office, ERP and MES Databases). In spite of the fact that each piece of equipment sold has a serial number, most of the time it takes a substantial amount of time to compile necessary documentation and data for the equipment. This documentation cannot be accessed on the web at the moment.

In a one-of-a-kind production environment or in an engineerto-order business model, usually a new business opportunity means a new project. Executing such an order involves specific combinations of different activities, such as understanding the customer's requirements, conducting a rough product design, feasibility studies, engineering, rough production planning, production, commissioning and after sales support. These processes are non-prescriptive; there is a lot of flexibility involved and many decisions are to be made by humans. 


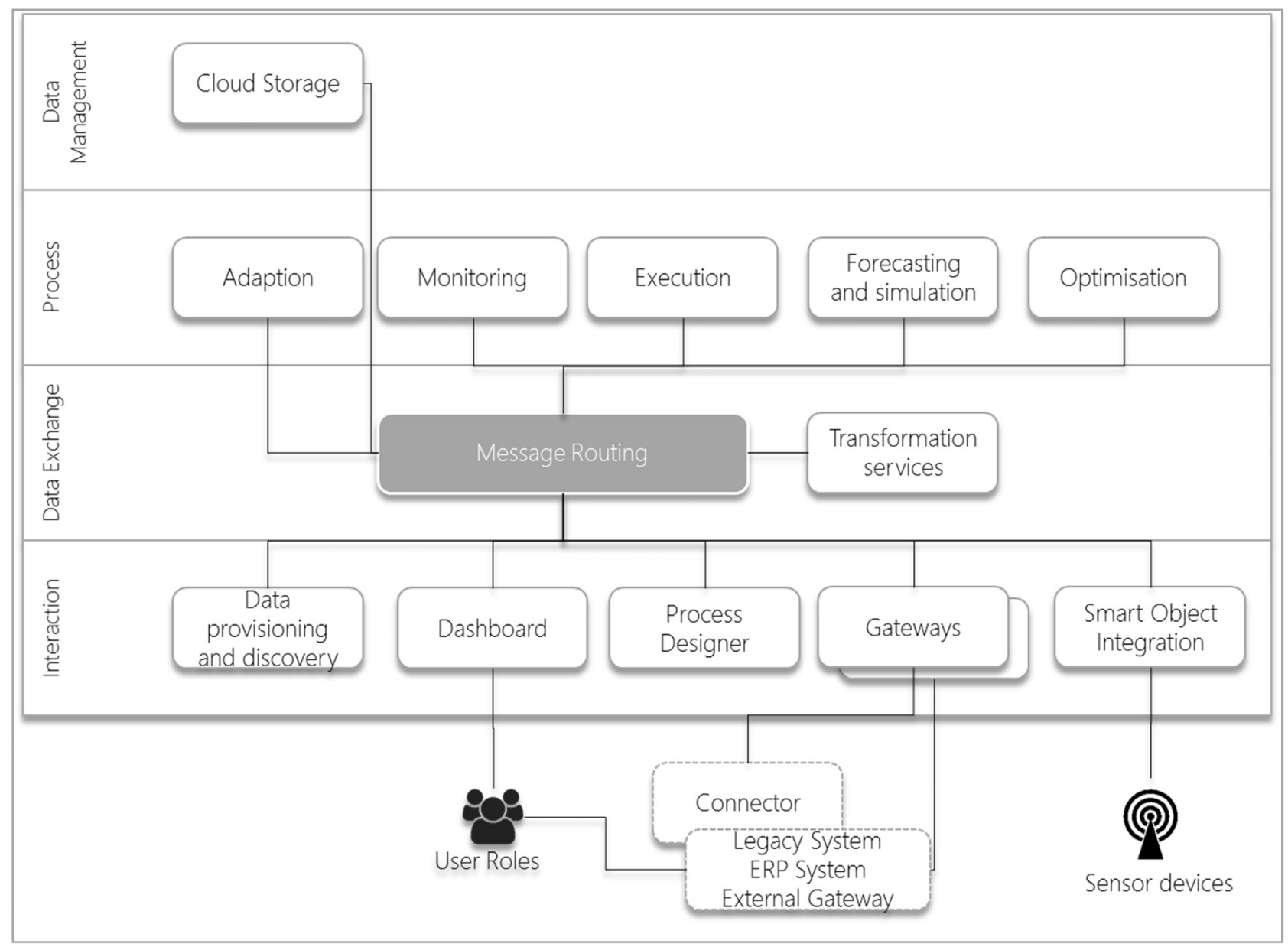

Fig. 6. Overall architecture of the platform.

In order to understand the applicability of the envisioned framework to this kind of environment, the business needs and requirements have been analysed using the reference model introduced in Section 4. This analysis led to the process model represented in Fig. 7.

As stressed before, this process includes engineering and manufacturing tasks. Both engineering and manufacturing tasks can be performed internally or outsourced. The outsourcing is decided by the industrial manager for each particular customer order. The manager takes into account the current and future production capacity available.

Since the engineering process involves product design activities, the processes of the extended enterprise cannot be fully designed a priori, as will happen in a conventional process management workflow suite. The design of the extended enterprise takes as input a process template providing a rough model that is likely to be changed several times along the life-cycle using the Process Designer tool. The model is progressively refined and specialised, and engineering proceeds with product design. The process model may also change during the execution time (adaption) in order to react to events, such as order delays or quality test failures.

The handling of each customer order includes a specific arrangement of intra and inter-enterprise tasks whose sequence is described by the process model developed. The management of these tasks will always involve a combination of automatic (legacy systems interfaces) and human-based decisions (using web and mobile interfaces). For example, internal orders can be dispatched automatically to the legacy systems. Moreover, assigning or re- assigning orders to suppliers or process re-planning will always involve human decisions. The life-cycle of each supplier order involves a set of negotiation, contracting, design or manufacturing tasks, expediting and in-bound tasks, as well as testing and verification tasks.

Using the process designer and the integration with the Profile Manager functional component (data provisioning and discovery), the process manager is able to search for suitable partners, (filtering them by non-functional requirements, such as $\mathrm{CO}_{2}$ emissions, lead-time, cost, location) and assign them to each external order. Once the process is defined, the simulation and optimisation components can be employed in order to achieve the best result for this concrete process.

The next step is the execution of the process (this is where the Smart Process Engine comes in), controlling the workflow and invoking services on the internal legacy systems, as well as on the legacy systems of the partners also involved in the collaboration network.

Gateways play an important role on Integration and interoperability, based on the SOA architecture. They are composed of standard components and custom components with functionality developed or created for connecting to a specific external system type and/or instance. Its mission is to communicate with a specific system, via manufacturing services available at the services repository. This means that a significant part of a gateway implementation is tailored to specific (e.g. SAP ERP) technology or communication/interface protocol. Gateways have an agnostic view of what is the format and the content exchanged. The information collected through the gateways will be verified and 


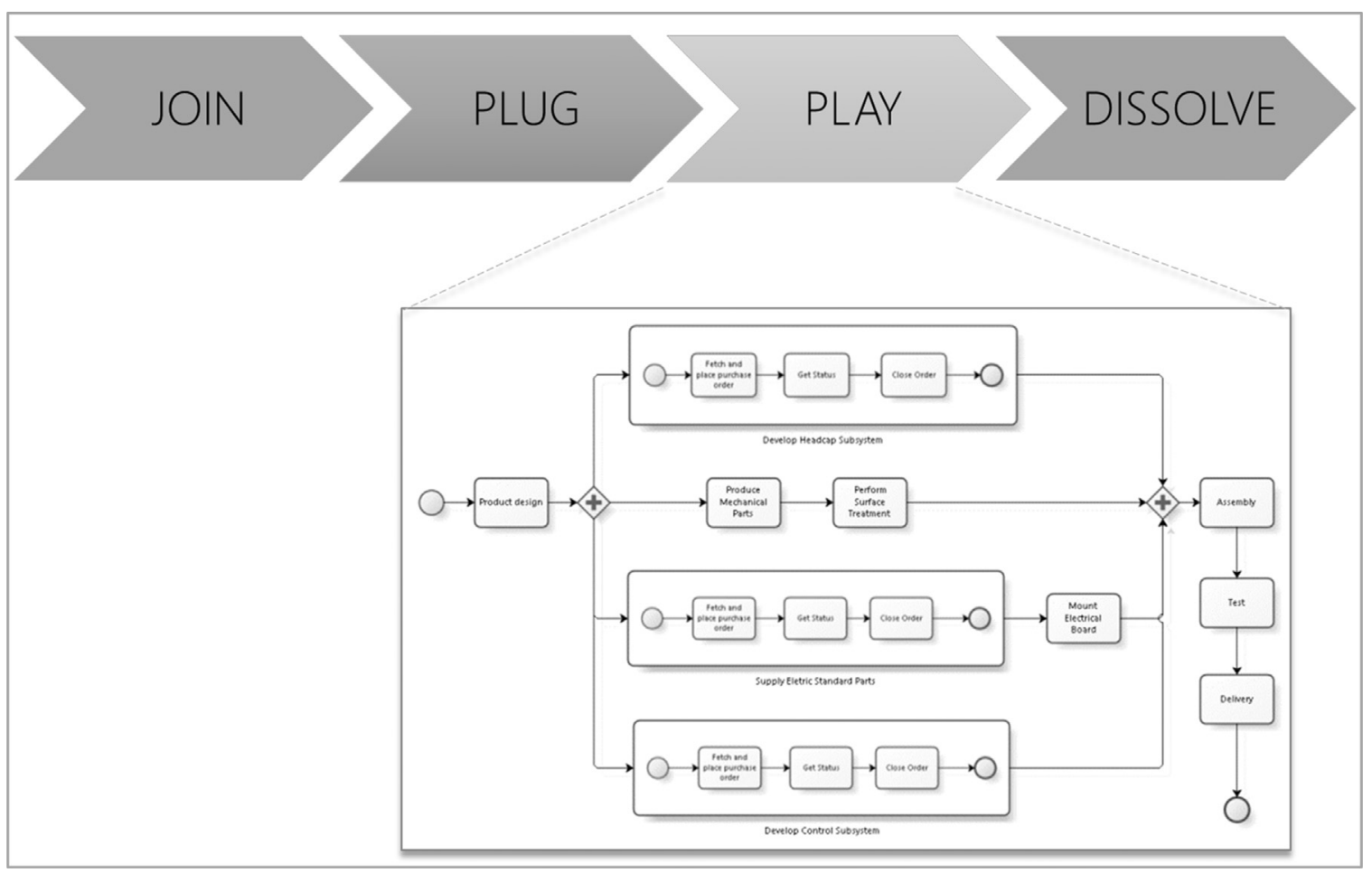

Fig. 7. The Play sub-process process model.

processed at the destination components (for instance, the Process Execution component). Gateways make no decisions themselves. However, gateways can call Transformation Services directly to translate from the external format to predefined (common) formats which are specified.

Main interrelations of gateway elements are related to:

\subsection{Dashboard}

Through the company configuration functionalities, the dashboard component exposes the user interfaces provided by this component (Fig. 8).

\subsection{Message routing}

Gateways interact with the message routing system in order to receive and deliver the information requested from/to the appropriate module; several components access the gateways this way (Fig. 9).

\subsection{Smart objects integration}

Gateways interact with the Smart Objects Integration component so that the information provided by the Smart Objects can be delivered to other components, and also allow these components to query the Smart Objects Integration component for specific data.

\subsection{Distributed Storage}

The descriptions of the gateway services and associated attachments will be stored using the Distributed Storage component.

To provide some examples, the following services have been implemented and registered at the manufacturing services repository, so the existing ERP can be integrated in the extended enterprise framework.

\subsection{1. submitOrder}

Submits an order to be manufactured by the factory.

\subsection{2. getOrderInfo}

Retrieves accounting and logistics information related to a submitted order.

\subsection{3. getOrderExecutionInfo}

Returns a description of the current state of the execution of a particular order, which contains data such as completion percentage, time to conclude the order and time to ship it.

\subsection{4. getProductExecutionInfo}

Returns a description of the current state of the execution of a particular product within an order; contains completion percentage and list of incidents.

\subsection{5. getProductInfo}

Returns factory capacity parameters related to the given product. Properties such as maximum capacity, unit price, or carbon footprint, can be obtained from the result.

\subsubsection{InformAdventureOnOrderEvent}

This operation functions as a push action that is invoked on the client side once a change occurs in the order production. For example, it will be called to notify about the completion of an order, or a new incident.

In this paper, only the lay phase was considered to illustrate the approach. Detailed information on the other phases of the lifecycle may be found in the technical reports of the ADVENTURE project, such as [14] Fig. 10.

\section{Conclusions and further research}

Today's business is characterised by a competition with dynamic and shorter market opportunities. In order to cope with 


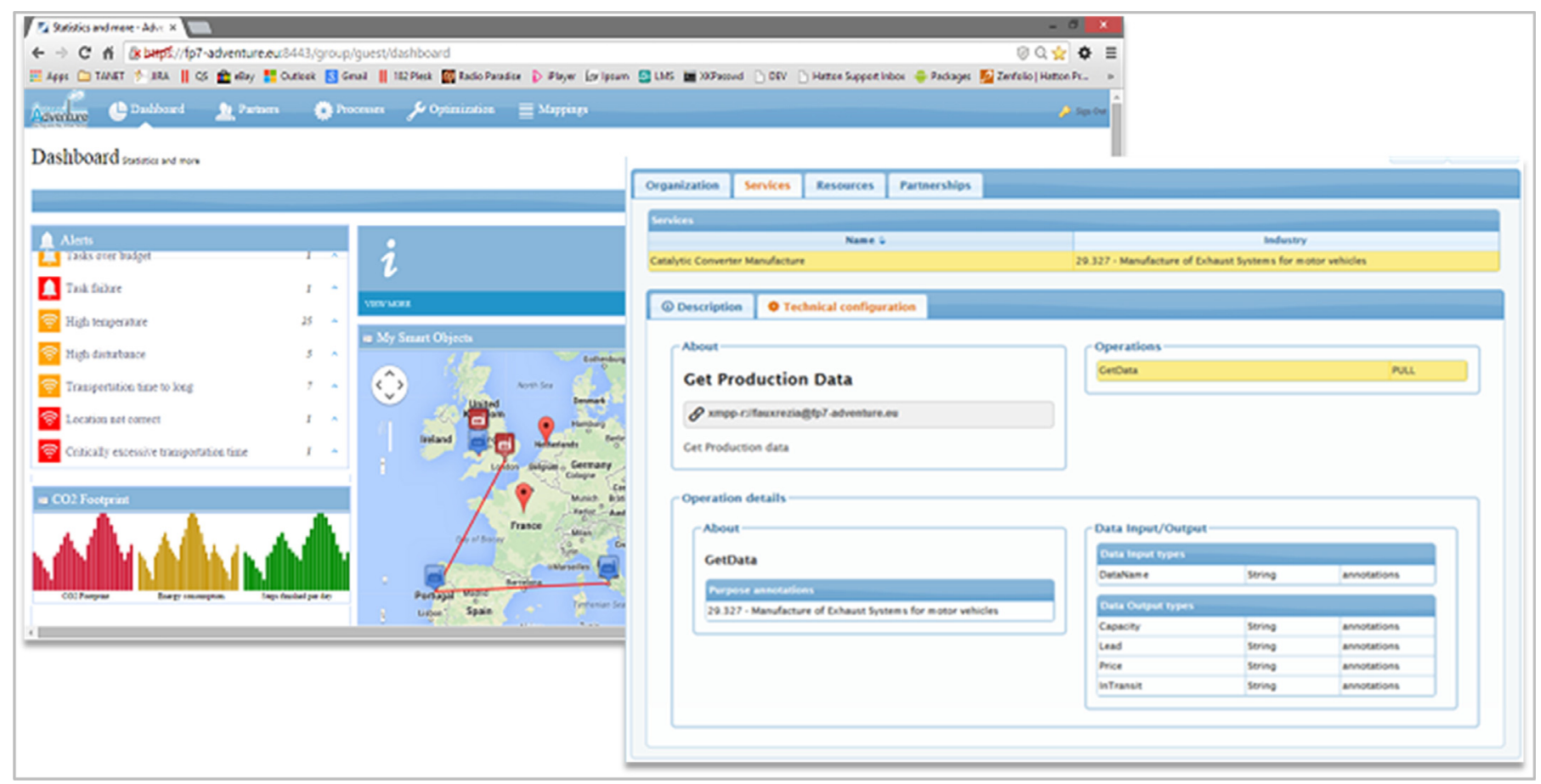

Fig. 8. Dashboard screenshot.

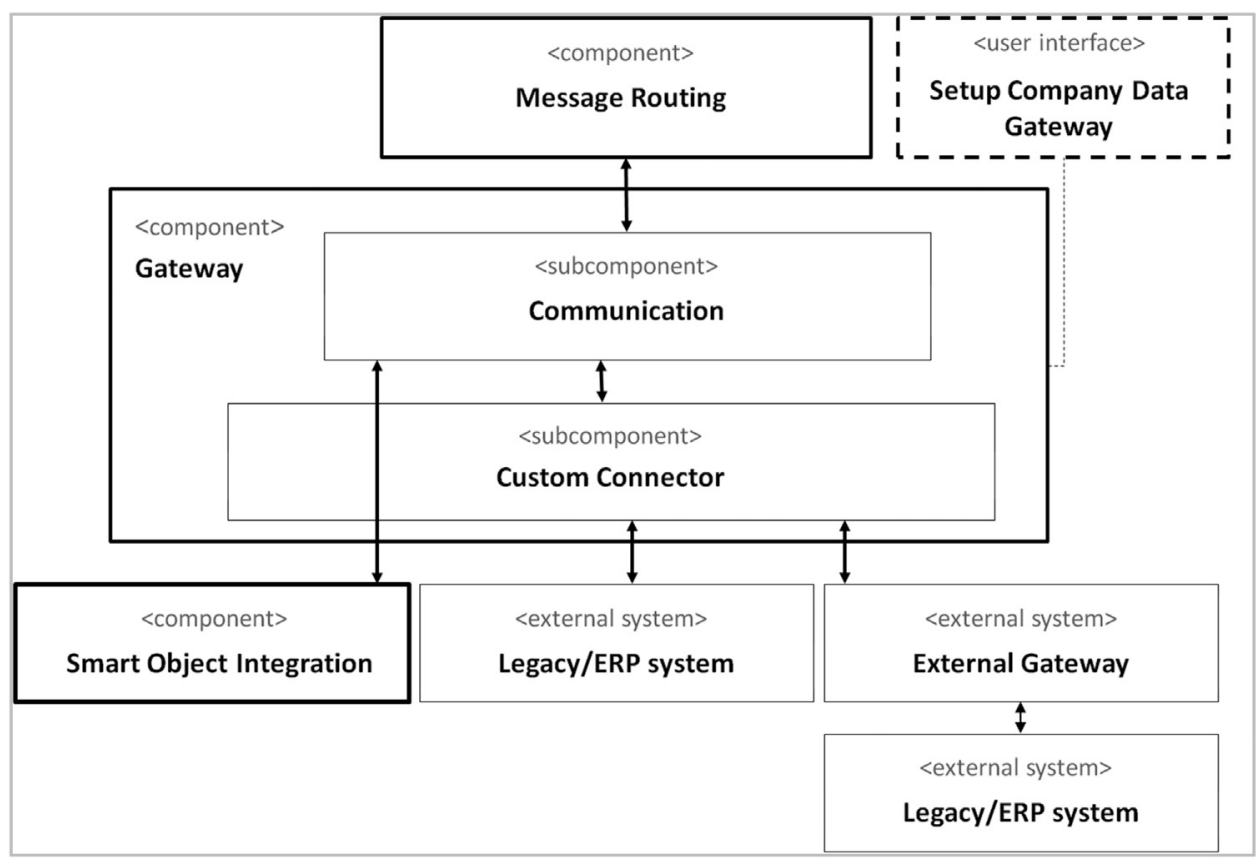

Fig. 9. Gateways general components diagram.

such environment, manufacturing firms, especially small and medium enterprises (SMEs), need to collaborate with each other for mutual benefits. It was in this context that the concept of extended enterprise emerged.

The operation of such enterprises can be compared with the concept of 'plug-and-play', where various business processes involving different companies are designed, validated and executed according to a specific need. During the plug phase, specific business requirements collected previously are integrated in the process design phase. In the play phase, predefined processes are adapted in order to run the extended enterprise successfully.

The ADVENTURE framework presented aims to provide SMEs with methodological and technical tools to simplify the establishment, management, adaptation, and monitoring of dynamic manufacturing processes in extended manufacturing enterprises, by building on concepts and methods from the field of serviceoriented computing. This framework allows enterprises to go beyond existing operational limitations by providing concrete tools and approaches for leveraging the information exchange between factories [4].

Most notably, it allows for integration with third-parties, that is, suppliers and customers, and facilitates a structured exchange of information. Accordingly, the platform serves as primary channel of communication, thus replacing the multiple - not 


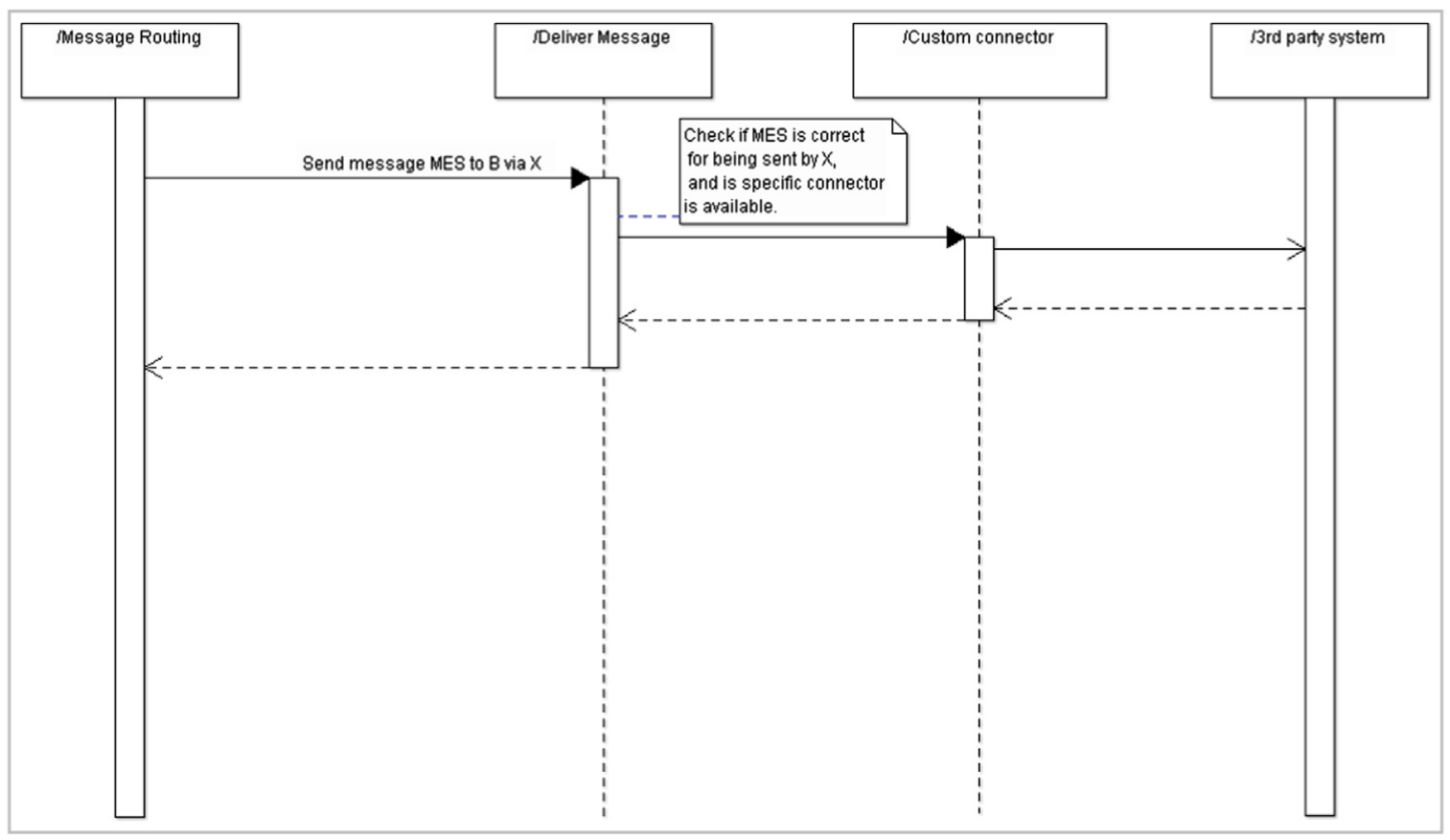

Fig. 10. Gateways general sequence diagram.

necessarily efficient - channels used today. In addition, it provides centralised information storage and allows data from various sources to be aggregated seamlessly.

However, to meet some specific requirements of a one-of-akind and engineer-to-order production environment, some improvements should be addressed, namely integration with content management systems and human-based decision. Furthermore, in the next steps, the framework should be validated in different application domains based on adaptive and collaborative manufacturing environments.

Likewise, increasing the supply chain visibility is important to all partners involved. This concerns aspects such as crowdsourcing, information exchange, real-time monitoring and status reports. Receiving important status information in a timely manner is important to all business partners, because it enables proactive rather than reactive - responses to potential problems in the supply chain.

The business case presented shows that the ADVENTURE framework fits almost all the requirements needed to manage those collaborative manufacturing processes.

\section{Acknowledgements}

This work was developed in the scope of the Project "TEC4Growth - Pervasive Intelligence, Enhancers and Proofs of Concept with Industrial Impact/NORTE-01-0145-FEDER-000020" which is financed by the North Portugal Regional Operational Programme (NORTE 2020), under the PORTUGAL 2020 Partnership Agreement, and through the European Regional Development Fund (ERDF).

\section{References}

[1] F. Jovane, E. Westkamper., D. Williams, The Manufuture Road: Towards Competitive and Sustainable High-Adding-Value Manufacturing, Springer-Verlag, Berlin Heidelberg, 2009.

[2] A. Azevedo, A. Almeida, Factory templates for digital factories framework, Robot.
Comput. Integr. Manuf. 27 (2011) 755-771.

[3] Luis Carneiro, et al., An innovative framework supporting SME networks for complex product manufacturing. Collaborative Networks for a Sustainable World, 336, 2010, pp. 204-211.

[4] ADVENTURE: D2.1 Project Vision Consensus Document. Deliverable D.2.1 of Adventure Project - ADaptive Virtual ENterprise ManufacTURing Environment FoF-ICT2011.7.3 - 285220, 2011.

[5] L.M. Camarinha-Matos, et al., Collaborative networked organizations - Concepts and practice in manufacturing enterprises, Comput. Ind. Eng. (2009).

[6] L.M. Camarinha-Matos, H. Afsarmanesh, Collaborative Networks -Reference Modeling, Springer, New York, 2008.

[7] M. Adams, et. al.: Worklets: A Service-Oriented Implementation of Dynamic Flexibility in Workflows. in: Proceedings of 14th International Conference on Cooperative Information Systems (CoopIS'06), Springer, 2006, pp. 291-308.

[8] V. Barba-Sánchez, M.P. Martínez-Ruiz, A.I. Jimenez-Zarco, Drivers, benefits and challenges of ICT adoption by small and medium sized enterprises (SMEs): a literature review, Probl. Perspect. Manag. 5 (1) (2007) 104-115.

[9] J. Faria, J. Silva, H. Marques, Supporting the Operation of Semi-Structured Work Systems, IN: Proceeding of CENTERIS 2010 - Conference on Enterprise Information Systems, Viana do Castelo - Portugal, 2010.

[10] M. Wesk, Business Process Management - Concepts, Languages, Architectures, Springer, 2007.

[12] SCC: SCOR - Supply-Chain Operations Reference Model Supply-Chain Council, 2011.

[13] VCG: VRM - Value Reference Model. Value Chain Group, 2010.

[14] ADVENTURE: D3.4 Conceptual Manufacturing Reference of Adventure Project ADaptive Virtual ENterprise ManufacTURing Environment FoF-ICT-2011.7.3 285220, 2011.

[15] Yang Cheng, Sami Farooq, John Johansen, Manufacturing network evolution: a manufacturing plant perspective, Int. J. Oper. Prod. Manag. 31 (12) (2011) $1311-1331$.

[16] M.T. Martinez, P. Fouletier, K.H. Park, J. Favrel, Virtual enterprise - organisation, evolution and control December 2001, Int. J. Prod. Econ. 74 (1-3) (2001) 225-238.

[17] Frank Meisela, Christian Bierwirth, The design of make-to-order supply networks under uncertainties using simulation and optimisation, Int. J. Prod. Res. 52 (22) (2014).

[18] Tianyi Pan, Zhi-Hai Zhang, Hui Cao, Collaborative production planning with production time windows and order splitting in make-to-order manufacturing, Comput. Ind. Eng. 67 (2014) 1-9.

[19] Hasse Nylund, Paul H. Andersson, Framework for extended digital manufacturing systems, Int. J. Comput. Integr. Manuf. 24 (5) (2011) 446-456.

[20] Xu, Li Da, Enterprise systems: state-of-the-art and future trends. Industrial Informatics, in: IEEE Transactions on 7.4, 2011, pp. 630-640.

[21] Michael P. Papazoglou, et al., Service-oriented computing: state of the art and research challenges, Computer 11 (2007) 38-45.

[22] Minglun Ren, J. Lyytinen Kalle, Building enterprise architecture agility and sustenance with SOA, Commun. Assoc. Inf. Syst. 22.1 (2008) 4

[23] Mohsen Mohammadi, Muriati Mukhtar., A review of SOA modeling approaches for enterprise information systems, Procedia Technol. 11 (2013) 794-800.

[24] State of the Art in Service-oriented Architecture, VDM Verlag Saarbrücken, Germany, ISBN $38364622659783836462266,2008$. 